


\section{resumo}

Este artigo visa a verificar o impacto da mudança nas condições de existência material (sanitárias e econômicas) causada pela pandemia de covid-19 na estabilidade dos valores materialistas e pós-materialistas no Brasil. A hipótese, construída a partir da literatura e testada com os dados de duas ondas do projeto "Valores em Crise", é que tais valores deveriam ser estáveis na vida dos indivíduos e não mudariam no curto prazo. Porém, os resultados indicam o oposto: um aumento dos valores pósmaterialistas entre os brasileiros em comparação com os materialistas. Dessa forma, o artigo trata da variação de diferentes tipos de valores e preferências sociopolíticas frente à crise e propõe explicações para as mudanças de curto prazo observadas, bem como as suas potenciais consequências no médio e no longo prazo.

Palavras-chave: pós-materialismo; pandemia; estabilidade de valores; projeto "Valores em Crise"; World Values Survey; Brasil.

\section{abstract}

This article aims to verify the impact of the change in material existence conditions (health and economic) caused by the covid-19 pandemic on the stability of materialistic and post-materialistic values in Brazil. The hypothesis, built from the literature and tested with data from two waves of the "Values in Crisis" project, is that such values should be stable in the lives of individuals and would not change in the short run. However, the results indicate the opposite: an increase in post-materialistic values among Brazilians compared to materialistic ones. Thus, the article deals with the variation of different types of values and socio-political preferences in the face of the crisis and proposes explanations for the observed short-term changes, as well as their potential consequences in the medium and long term.

Keywords: post-materialism; pandemic; value stability; "Values in Crisis" project; World Values Survey; Brazil. 


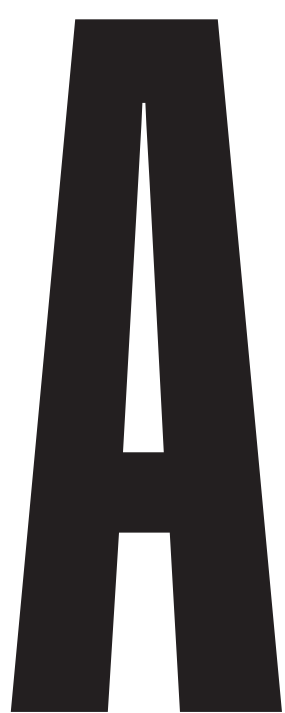

crise sanitária e econômica provocada pela pandemia de covid-19 afetou severamente os países latino-americanos, principalmente o Brasil. Com mais de 576 mil mortos até setembro de 2021 e uma variante própria do vírus, o país é apontado como um risco epidemiológico para a região por organismos multilaterais e a comunidade científica internacional (The Lancet, 2020). Os brasileiros têm sentido o aumento dos níveis de insegurança existencial no país, por perdas humanas e sequelas após a contração da doença, por falta de clareza sobre medidas que evitem a propagação do vírus ou promovam o seu combate (Da Silva \& Ventura, 2020) e por perdas de emprego e redução da renda (Barbosa, Costa \& Hecksher, 2020), o que tem aprofundado as desigualdades e as vulnerabilidades de diversos setores da população (Pires, 2020). Além disso, as respostas oficiais à crise têm estimulado o negacionismo e a desinformação, dentro dos padrões de polarização política preexistentes no país (Vizcarra Castillo, Santos \& Castro, 2020). A gravidade da situação levou diversos pesquisadores a apontarem o país como um pária em termos de saúde global (Ventura \& Costa Bueno, 2021; Werneck \& Carvalho, 2020). Tanto na academia como na esfera política, diversos questionamentos sobre as responsabilidades desta crise sem precedentes no país têm sido feitos.

Paradoxalmente, a crise decorrente da pandemia se constitui em momento sui generis para o estudo dos efeitos que fenômenos externos críticos, que mudam profundamente a dinâmica social, têm sobre as atitudes e valores dos indivíduos (Esaiasson et al., 2020;

HENRIQUE CARLOS DE O. DE CASTRO é professor no Programa de Pós-Graduação em Ciência Política e do curso de Relações Internacionais da UFRGS.

DANIEL CAPISTRANO é pesquisador da Escola de Educação da University College Dublin.

SOFIA ISABEL VIZCARRA CASTILLO

é professora da Universidade do Vale do Rio dos Sinos (Unisinos). 
Mellon, Bailey \& Prosser, 2021; Prieto-Ursúa \& Jódar, 2020; Reeskens et al., 2021; Rothstein, 2020). Nesse sentido, e na linha apontada pela equipe de Reeskens (2021), este artigo propõe verificar em que medida a crise da covid-19 afeta a estabilidade de três dimensões da cultura e valores políticos dos brasileiros: i) valores básicos (por exemplo, religiosidade); ii) valores morais (aqui medidos segundo o índice de materialismo/pós-materialismo de Inglehart); e iii) atitudes sociopolíticas (por exemplo, o posicionamento ideológico). Nossa hipótese é que aqueles valores mais fundamentais, como valores básicos e morais, deveriam ser estáveis no tempo por serem adquiridos em processos de socialização de longa data (Inglehart, 1997). Assim, a partir da análise dos dados das duas ondas do projeto "Valores em Crise" (VIC) e da pesquisa World Values Survey (WVS), testamos a estabilidade destas três dimensões frente aos efeitos da pandemia. Entendemos que a mudança nas condições de existência material (sanitárias e econômicas) no curto prazo não deveriam impactar significativamente os valores morais, a cultura política ou os valores fundamentais, como a religiosidade. No entanto, as particularidades do Brasil no contexto da pandemia, principalmente o enquadramento discursivo e mediático da crise e os impactos econômicos e humanos, também devem ser consideradas para a compreensão da estabilidade ou mudança desses valores.

\section{ESTABILIDADE OU VOLATILIDADE DOS VALORES E PREFERÊNCIAS SOCIOPOLÍTICAS NO TEMPO}

A cultura política é caracterizada pela distribuição entre os cidadãos de um conjunto de crenças e atitudes em relação a determinados objetos políticos da sociedade (Almond \& Verba, 1989). Ela está configurada a partir de valores fundamentais, como, por exemplo, a religiosidade, porém também se manifesta nas escolhas e valorações sobre assuntos políticos. Nesse sentido, diferentes autores (Converse, 2006; Uslaner, 2002) estabeleceram uma hierarquia entre as diferentes preferências dos cidadãos, classificando-as com base no seu nível de volatilidade. Dito de outra forma, os valores fundamentais ou associados às condições de socialização da infância seriam mais estáveis na vida do indivíduo (é o caso dos valores materialismo e pós-materialismo de Inglehart, 1977). Já assuntos sobre a vida em sociedade e preferências avaliativas tenderiam a mudar segundo a conjuntura ou a forma como a informação é apresentada. Neste artigo, retomamos e adaptamos a escala proposta por Reeskens (2021) de volatilidade dos valores morais (Figura 1).

Uma situação como a pandemia de covid19 pode alterar a forma de preferências sobre assuntos específicos dada a urgência de decisões sobre determinados temas; precisamos, então, compreender se a gravidade da crise teria modificado em algum sentido a estabilidade dos valores morais. Momentos de crise na América Latina, como na Argentina em 2001 e em outros países da região no final do século $\mathrm{XX}$, causaram modificações da prevalência de valores pós-materialistas naquelas sociedades (Ribeiro \& Borba, 2010). Assim, a seguir explicamos a operacionalização das quatro dimensões da cultura política presentes na Figura 1 a partir dos dados das pesquisas WVS e VIC, bem como as hipóteses teóricas sobre sua estabilidade e os resultados com relação às mudanças de cada dimensão. 


\title{
FIGURA 1
}

\section{Estabilidade e volatilidade de dimensões da cultura política}

\section{Estável}

\author{
Valores básicos (ex.: religiosidade)
Valores morais (ex.: pós-materialismo)
Atitudes sociopolíticas (ex.: esquerda/direita)
Preferências avaliativas (ex.: políticas públicas/confiança)
}

Volátil

Fonte: adaptado com base em Reeskens et al. (2021)

\section{MÉTODOS E RESULTADOS}

Para investigar a estabilidade dos valores pós-materialistas durante a pandemia, utilizamos os dados da pesquisa VIC. Essa pesquisa foi realizada em duas ondas: a primeira entre maio e junho de 2020 e a segunda onda entre janeiro e fevereiro de 2021, em formato de painel (mesmos entrevistados nas duas ondas). Indivíduos das cinco regiões do Brasil preencheram o questionário por meio de Entrevista Web Assistida por Computador (CAWI). A primeira onda da pesquisa contou com 3.543 respondentes, e a segunda, com 1.929 indivíduos, ou seja, 54\% da amostra inicial. O presente estudo utilizou somente os dados de respondentes que fizeram parte das duas ondas da pesquisa ${ }^{1}$.

O questionário contou com itens relacionados à percepção sobre a crise, além de

1 Analisamos também o perfil de respondentes que não participaram da segunda onda. Verificamos que a razão de chance de ausência na segunda onda é menor entre as mulheres respondentes mais jovens, de classes sociais mais altas e de maior escolaridade. questões sobre valores e atitudes políticas. Alguns desses itens foram reproduzidos do questionário da pesquisa WVS, implementada por um consórcio de cientistas sociais de vários países do globo. O seu questionário tem cerca de 250 questões e é aplicado em entrevistas face a face em amostras nacionais representativas. É realizada desde a década de 1980 e repetida a cada cinco ou sete anos em mais de 100 países, compreendendo cerca de $80 \%$ da população mundial. O Brasil participou de cinco pesquisas da WVS, sendo a última realizada no ano de 2018.

Os dados da pesquisa VIC não são diretamente comparáveis aos dados da WVS devido a diferenças de metodologia e de composição da amostra. Contudo, ao longo desta análise, fazemos referência aos dados da WVS para incluir uma perspectiva histórica às reflexões apresentadas.

A análise foi dividida em duas partes: 1) descrição dos itens que representam cada uma das dimensões da cultura política e as nossas expectativas em relação à mudança ou permanência de padrão durante a pandemia; 2) verificação de eventual mudança signifi- 
cativa de cada um desses itens da primeira para a segunda onda da pesquisa VIC.

\section{Valores religiosos}

Desde Karl Marx e Max Weber, diversas teorias da modernização enfatizam o papel das mudanças de valores religiosos e de um processo de secularização observado em várias sociedades nos últimos séculos. No entanto, existe um certo consenso entre cientistas sociais acerca da permanência desses valores ao longo de curtos períodos intrageracionais (Converse, 2006; Inglehart, 1997).

A pesquisa WVS possui diversas questões sobre religião, entre elas, o quão importante ela é na vida dos entrevistados. Os itens de resposta são "Nada importante", "Pouco importante", "Algo importante", ou "Muito importante". Em uma escala numérica em que 1 representa "Nada importante" e 4 denota "Muito importante", a média na amostra brasileira variou de 3,36 (Desvio Padrão² DP $=0,88)$ em 1991 até 3,25 (DP $=0,41)$ em 2018. Ou seja, além de haver uma alta prevalência de pessoas religiosas no país (médias próximas a quatro), a proporção não se alterou significativamente ao longo de quatro décadas.

A mesma pergunta foi incluída na pesquisa VIC, que registrou uma média similar à onda da WVS de 2018: valor médio de $3,32(\mathrm{DP}=0,92)$ no ano de 2020 e de 3,27 $(\mathrm{DP}=0,93)$ em 2021. Apesar da redução no valor médio dessa escala de religiosidade de

2 Desvio Padrão (DP) é uma medida de dispersão: quanto maior for o DP, maior a dispersão de dados, ou seja, as respostas dos entrevistados assumem valores muito diferentes; quanto menor, menor a variabilidade de valores.
2020 para 2021, de acordo com a literatura e como hipótese deste trabalho, acreditamos que essa diferença entre as ondas não seja significativa, ou seja, tal redução se deve a algum fator aleatório.

A mudança de valores religiosos na pesquisa VIC incluiu práticas religiosas. Para tal, foi considerada a seguinte questão: "Com exceção de casamentos e funerais, com mais ou menos qual frequência você normalmente participava de atividades religiosas antes da crise do coronavírus?"; e, na segunda onda, "Com qual frequência participou dessas atividades desde o início da crise do coronavírus?". As categorias de resposta vão de "Nunca ou praticamente nunca" até "Mais de uma vez por semana" em uma escala de sete pontos. A média variou de 3,75 (DP = $0,5)$ na primeira onda para $3,73(\mathrm{DP}=0,52)$ na segunda onda. Nesse sentido, esperamos que a estabilidade dos resultados com relação à religiosidade sirva como parâmetro para as mudanças nos valores morais.

\section{Valores morais}

De acordo com Inglehart (1997, 2016), o pós-materialismo é uma síndrome de mudança de valores morais resultante de uma crescente segurança física e existencial que indivíduos experimentam a partir do desenvolvimento econômico e social. No entanto, essa mudança de valores, argumenta Inglehart, se dá de maneira lenta, gradual e geracional, uma vez que esses valores são adquiridos no momento de socialização e não tendem a mudar ao longo da vida (Inglehart, 1997, 2016; Taniguchi, 2006). Embora existam evidências de que valores morais possam se alterar ao longo da vida (Tormos, 2012, 2019), não esperamos que o nível de pós-materialismo se altere 
no curto período de tempo entre as duas ondas da pesquisa VIC, seguindo a teoria do pós-materialismo revisitado.

A mensuração de valores pós-materialistas nessa pesquisa se deu por meio da seguinte questão: "As pessoas às vezes falam sobre quais metas este país deveria ter para os próximos dez anos. Abaixo listamos algumas que receberiam prioridade máxima por diferentes pessoas. Por favor, indique qual a ordem de importância de cada uma delas, sendo 1 a mais importante e 4 a menos importante".

As categorias de resposta oferecidas foram "Manter a ordem na nação", "Dar mais voz às pessoas na política", "Combater a inflação" e "Proteger a liberdade de expressão". De acordo com a teoria de Inglehart, de todas as combinações possíveis, a seleção do par "Manter a ordem na nação" e "Combater a inflação" como mais importante indicaria que o respondente teria valores mais "materialistas". A seleção do par "Dar mais voz às pessoas na política" e "Proteger a liberdade de expressão" indicaria valores mais pós- -materialistas. A seleção de qualquer outro par de alternativas como mais importante indicaria uma posição mista.

A proporção de pós-materialistas manteve-se estável de 1991 a 2020, incluindo a primeira onda da pesquisa VIC. No entanto, conforme apontado na Figura 2, esse porcentual aumentou consideravelmente de cerca de $10 \%$, em 2018 e 2020, para $22 \%$ na segunda onda, em 2021.

Também medimos o pós-materialismo por meio de uma escala de quatro pontos, em que o valor mais alto representa a escolha das duas respostas pós-materialistas e o valor mais baixo, as duas respostas materialistas (Reeskens et al., 2021). A média dessa escala também aumentou de 2,0 (DP = 1) na primeira onda para $2,14(\mathrm{DP}=1,1)$ na segunda onda.

\section{Atitudes sociopolíticas}

Tal como os valores morais, a literatura também enfatiza a relativa estabilidade de ideologia e valores políticos. No entanto,

\section{$\begin{array}{ll}\bar{\equiv} & \text { FIGURA 2 }\end{array}$}

Porcentual de pós-materialistas na amostra brasileira, 1991-2021

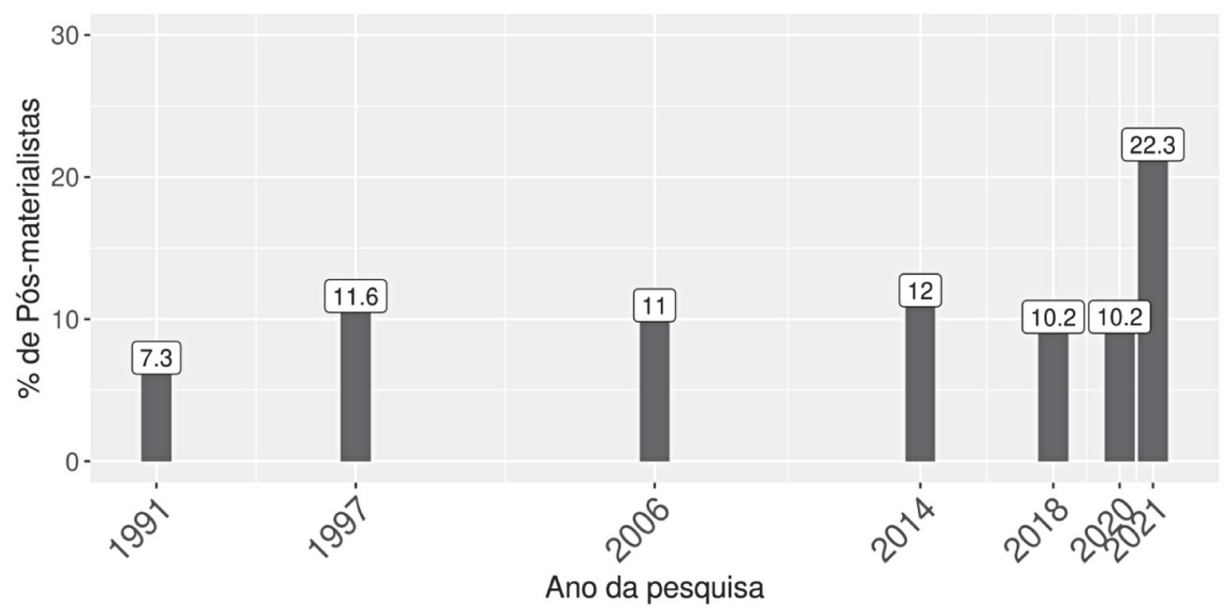

Fonte: Elaboração própria a partir dos dados das pesquisas WVS e VIC 
ao contrário da discussão sobre pós-materialismo, ideologias e orientações políticas podem ser influenciadas por contextos políticos e experiências individuais de curto e médio prazos e apresentarem maior volatilidade comparadas com valores morais.

Para medir ideologia política, utilizamos a escala de autoposicionamento político esquerda/direita. A mesma escala é utilizada nas duas pesquisas: o respondente é solicitado a escolher um ponto em uma escala em que 1 representa "Esquerda" e 10 representa "Direita". O valor médio dessa escala foi de 5,45 em 1991, passando a 5,90 em 1997 e a 5,41 em 2018. Na primeira onda da pesquisa VIC, o valor médio registrado foi de 5,98 e se manteve estável na segunda onda com 5,96 . Esse aumento na escala observado entre 2018 e 2020 pode ser associado ao aumento do conservadorismo de direita no Brasil discutido por diversos autores (Menéndez \& González, 2019; Pinheiro-Machado et al., 2019; Santos \& Tanscheit, 2019; González, Baquero \& Grohmann, 2020).

Juntamente com ideologia política, a visão política sobre igualdade de gênero pode assumir níveis similares de estabilidade e volatilidade. Na WVS, há a seguinte afirmação: "Quando os empregos estão escassos, os homens deveriam ter mais direito de conseguir um emprego do que as mulheres". O porcentual de respondentes que concordam com essa afirmação variou de 38,7\% em 1991 para $21,9 \%$ em 2006 e 19,1\% em 2018. A mesma questão foi incluída na pesquisa VIC, com um porcentual de concordância de $8,9 \%$ em 2020 e de $9,9 \%$ em 2021. Essa diferença entre os porcentuais aferidos pela WVS e VIC pode ser explicada pelo viés de participação no painel VIC discutido acima, que indica uma sobrerrepresentação de mulhe- res, de pessoas com maior escolaridade e de classes sociais mais altas.

Dois aspectos adicionais se destacam nessa dimensão, ambos relacionados à coesão social. Primeiramente, com vistas a avaliar a propensão à solidariedade, os respondentes foram perguntados em que medida se preocupam com o bem-estar de "Pessoas desconhecidas de sua cidade" e de "Pessoas desconhecidas de outros lugares (cidades, estados, países)". Outra questão relevante é se a maioria das pessoas iria tirar vantagem se tivessem a oportunidade ou se buscariam ser justas.

Por fim, outro aspecto relacionado às atitudes sociopolíticas é o grau de orgulho nacional. Na WVS, foi perguntado em que medida o respondente se sente orgulhoso de ser brasileiro. Em uma escala que varia de 1 a 4, com valores mais altos indicando maior orgulho, observa-se uma redução do nível de orgulho nacional ao longo de quatro décadas. As médias mais altas dessa escala foram verificadas em 1991 (3,44) e 1997 $(3,46)$. Esse valor médio diminui constantemente até chegar a 2,78 em 2018. A mesma questão incluída na pesquisa de 2020 indica um valor médio de 2,83 , que se mantém constante em $2021(2,84)$.

Dessa forma, apesar de mais voláteis do que valores religiosos e morais, valores sociopolíticos tendem a mudar somente no longo prazo. Assim, não são esperadas mudanças significativas de um ano para o outro a despeito da influência da pandemia.

\section{Mudança e permanência da cultura política ao longo da pandemia}

Para avaliar a estabilidade ou volatilidade das referidas dimensões da cultura política 
ao longo da pandemia, adotamos a estratégia metodológica empregada por Reeskens et al. (2021). Primeiramente, avaliamos o grau de concordância da posição individual na primeira onda comparada com a segunda onda. Para isso, utilizamos a estatística Tau-b derivada do coeficiente de correlação de Kendall (Uslaner, 2002). Tal estatística indica o grau de concordância a partir de uma escala que varia entre 0 e 1 , sendo 1 o nível mais alto de concordância entre as duas ondas.

Além disso, utilizamos um Teste $\mathrm{T}$ para avaliar se a média observada na primeira onda é significativamente diferente da média observada na segunda onda. A Tabela 1 apresenta o resultado ordenado pelo grau de correlação entre as duas ondas.

A maioria dos resultados de mudança ou estabilidade segue o padrão encontrado na literatura. Conforme esperado, as variáveis com o maior grau de correlação, indi- cando estabilidade, são relacionadas a valores religiosos. De maneira similar, não foram verificadas diferenças significativas entre as duas ondas da pesquisa no que tange a atitudes sociopolíticas (esquerda/direita, orgulho nacional, igualdade de gênero). Também em concordância com as hipóteses levantadas, preferências de políticas públicas de imigração e cooperação internacional experimentaram mudanças significativas com baixo grau de correlação entre as respostas indicadas na primeira e segunda ondas.

Contudo, alguns resultados apontam para padrões de mudança ou estabilidade distintos do esperado. A confiança no setor de saúde e no governo manteve-se inalterada apesar do forte impacto político ocasionado pela pandemia. Ainda assim, a hipótese sugerida é de que a confiança institucional é mais suscetível a mudanças e não necessariamente será alterada em virtude de uma mudança no

\section{TABELA 1}

Estabilidade e volatilidade de aspectos da cultura política

\begin{tabular}{llcll} 
Variável & Total & Intervalo & Tau-b & Valor-t* \\
\hline Religião é importante & 1929 & $1-4$ & 0.640 & -1.52 \\
\hline Prática religiosa & 1929 & $2-4$ & 0.588 & -1.49 \\
\hline Orgulho nacional & 1911 & $1-4$ & 0.574 & 0.28 \\
\hline Confiança no governo & 1929 & $1-4$ & 0.555 & 0.1 \\
\hline Esquerda/direita & 1929 & $1-10$ & 0.537 & -0.28 \\
\hline Igualdade de gênero & 1929 & $1-4$ & 0.382 & 0.19 \\
\hline Política imigratória aberta & 1929 & $1-4$ & 0.374 & $3.21^{* *}$ \\
\hline Pós-materialismo & 1929 & $1-4$ & 0.341 & $3.38^{* * *}$ \\
\hline Confia no setor de saúde & 1929 & $1-4$ & 0.334 & 2.01 \\
\hline Solidariedade & 1929 & $1-5$ & 0.293 & -1.31 \\
\hline Pessoas seriam justas & 1929 & $1-10$ & 0.284 & -0.71 \\
\hline Cooperação internacional & 1929 & $1-10$ & 0.252 & $4.3^{* * *}$ \\
\hline *significant at $\mathrm{p}<0.01,{ }^{* *}$ sig. at $\mathrm{p}<0.005, * * *$ sig. at $\mathrm{p}<0.001$ & \\
\hline
\end{tabular}


contexto sociopolítico. Por outro lado, não era esperado que as posições individuais sobre o pós-materialismo se alterassem em um curto prazo. De acordo com a teoria do pós-materialismo, esses valores morais são adquiridos no momento de socialização e não estão sujeitos a mudanças em curto prazo. Ainda que se assumisse como justificativa que a pandemia causou impactos sem precedentes à segurança física e existencial dos indivíduos, a direção da mudança esperada seria contrária, de diminuição do pós-materialismo e não de aumento, como foi verificado.

\section{DISCUSSÕES}

Frente a esses resultados, e visto que uma das dimensões com mudanças mais significativas foi o pós-materialismo, é pertinente discutir brevemente as suas características na América Latina, onde a prevalência dos valores pós-materialistas é incipiente em comparação com outras regiões do mundo. Segundo a teoria revisitada da modernização de Inglehart e Welzel (Inglehart, 1977; Inglehart \& Welzel, 2005), as melhorias das condições socioeconômicas das sociedades e, portanto, a redução dos níveis de insegurança existencial, atingidas através do aumento dos níveis de desenvolvimento, levariam a uma lenta mudança nos valores morais dos indivíduos. Esses valores passariam de uma orientação materialista tradicional (valorando a ordem, autoridade e a família) para uma orientação pós-materialista, com ênfase na liberdade, autoexpressão e realização pessoal. As revisões da teoria mostram que, apesar de se verificarem estatisticamente mudanças no aumento da prevalência do pós-materialismo, essa mudança não segue um processo linear e deve ser compreendida no contexto de cada sociedade, devendo também ser problematizada a sua relação com o fortalecimento do modelo de democracia liberal. Assim, por exemplo, no caso latino-americano, estudos mostram que o aumento moderado dos níveis de prevalência do pós-materialismo não impacta de maneira significativa os processos de fortalecimento da democracia e maior participação política, seja ela convencional (Castro \& Capistrano, 2008) ou não convencional (Barbosa et al., 2011; Ribeiro, Borba \& Hansen, 2016).

A análise desses valores na região, contudo, permite uma discussão sobre as formas em que os valores pós-materialistas se difundem e ganham maior prevalência. Assim, por exemplo, estudos anteriores (Castro, Ranincheski \& Capistrano, s. d.; Castro et al., 2020) explicam as variações nos valores pós-materialistas na América Latina em função da sua conexão com culturas globais e os efeitos da globalização. Nesse sentido, se entendermos a crise da pandemia como um momento de evidência crítica da nossa interdependência com a sociedade global, devemos ter em consideração essa característica para compreender os seus impactos na estabilidade dos valores morais. Considerando as características dos participantes na pesquisa VIC (maior acesso à internet e, portanto, maior acesso a canais de consumo da cultura global), as limitações de acesso aos encontros físicos e aos centros de difusão de valores globais (como shoppings) podem ter influenciado a sua conexão com o mundo global através de formas de consumo digitais.

A dimensão global está presente também na forma como foi abordada a pandemia no Brasil, com o aumento do apoio à cooperação internacional visto nos resultados. Assim, por exemplo, apesar de a crise ser tratada como 
um problema materialista (saúde vs. economia), as discussões sobre as suas potenciais soluções, veiculadas pela imprensa, pela academia, assim como nas ações dos governos estaduais, vistos como quem dava respostas concretas à crise, tinham uma dimensão internacional. A imprensa enfatizava o combate à desinformação disseminada em redes sociais e as diretrizes de organismos multilaterais como a Organização Mundial da Saúde (OMS) (Couto, Couto \& Cruz, 2020; Ferraz, 2020; Santos-Pinto, Miranda \& Osorio-de-Castro, 2021), enquanto as redes sociais e o próprio governo emitiam mensagens contrárias a tais diretrizes internacionais.

A postura isolacionista e negacionista do governo federal (Da Silva \& Ventura, 2020) também fica evidenciada na ação de articulação internacional realizada por diferentes governadores durante a pandemia (Alvarenga et al., 2020; Oliveira \& Nery, 2021; Rei, 2021). Assim, enquanto os governadores recorriam à cooperação internacional para obter insumos e vacinas, como foi o caso do Maranhão e São Paulo, o governo federal atrasava aprovações de compras de insumos essenciais e promovia medicamentos contraproducentes para a saúde pública, como o denominado "kit covid". Todas essas ações, em um público com acesso a uma diversidade de informações, podem levar a uma mudança na relação com a cooperação internacional, assim como valores de autoexpressão vinculados em certa medida com liberdade de imprensa.

No mesmo sentido, a confiança no setor de saúde reflete a confiança nas ações institucionais do setor. Com efeito, alguns autores (Costa, Rizzotto \& Lobato, 2020; Facchini, 2020) discutem a pandemia como uma oportunidade para enxergar a importância do SUS e resgatar o papel de lide- rança histórica do Brasil em questões de saúde global (Albuquerque, 2020; Ventura \& Costa Bueno, 2021).

\section{CONCLUSÕES}

A crise sanitária e econômica provocada pela pandemia de covid-19 afetou o mundo inteiro, mas de maneira especialmente severa os países da América Latina, particularmente o Brasil. Por diversas razões, o país está sendo apontado como um risco epidemiológico para a região e mesmo para o planeta por organismos multilaterais. Tal situação permite testar hipóteses encontradas na literatura especializada sobre a persistência ou mudança de determinados valores no curto prazo em função de fatores exógenos. Assim, este artigo visou a verificar em que medida a mudança nas condições de existência material das pessoas em função da crise sanitária e econômica advinda da pandemia, no curto prazo, impactou a estabilidade dos valores materialistas e pós-materialistas no Brasil. Utilizamos para tanto a análise dos dados das duas ondas do projeto "Valores em Crise" e do conjunto das ondas da pesquisa World Values Survey realizadas no Brasil.

A maioria dos resultados encontrados a partir da análise dos dados indica mudança ou estabilidade segundo o padrão encontrado na literatura. Conforme esperado, dados relacionados a valores religiosos se mantêm estáveis durante a pandemia. De maneira similar, não foram verificadas diferenças significativas em relação a atitudes sociopolíticas (esquerda/direita, orgulho nacional, igualdade de gênero). Ainda de acordo com o esperado, não foram verificadas mudanças significativas na opinião sobre políticas públicas de imigração e coo- 
peração internacional entre a primeira e a segunda onda da pesquisa VIC.

Alguns resultados, no entanto, apontam para padrões de mudança ou estabilidade distintos do esperado. Por um lado, a confiança na área da saúde e no governo não se modificaram, apesar do importante debate político e da omissão e negacionismo do governo federal durante a pandemia. Por outro, a alteração das posições individuais sobre o pós-materialismo em um tão curto prazo vai de encontro ao que diz a literatura e às nossas hipóteses iniciais. A teoria do pós-materialismo sustenta que valores morais, que são adquiridos no momento de socialização, não mudariam no curto prazo. Ainda que considerássemos todos os transtornos e medos causados pela crise advinda da pandemia e os seus impactos na segurança física e existencial dos indivíduos, a direção da mudança esperada seria contrária, de diminuição do pós-materialismo e não de aumento, como foi verificado.

Como constatado em outros trabalhos realizados anteriormente por nosso grupo de pesquisa, concluímos que a teoria do pós-materialismo revisitado precisa ser atualizada em função de novos achados. Uma hipótese que temos sustentado é que há a necessidade de ajustes na sua aplicação em países não centrais do sistema econômico e político do planeta, uma vez que há indícios de que os dados parecem indicar a sua melhor adequação aos países mais desenvolvidos, como os do norte da Europa. Dessa forma, acreditamos que este artigo possa contribuir com tal debate.

\section{REFERÊNCIAS}

ALBUQUeRQUE, M. “Globalização da Saúde Pública: a Organização Mundial da Saúde e a cooperação na América do Sul". PrIntCAPES, n. 1, 2020.

ALMOND, G. A.; VERBA, S. The civic culture: political attitudes and democracy in five nations. California, SAGE Publications, 1989.

ALVARENGA, A. A. et al. "Challenges for the Brazilian state from the covid-19 pandemic: the case of paradiplomacy in the state of Maranhão". Cadernos de Saúde Pública, v. 36, n. 12, 2020.

BARBOSA, A. L. N. de H.; COSTA, J. S.; HECKSHER, M. "Mercado de trabalho e pandemia da covid-19: ampliação de desigualdades já existentes?". Boletim Mercado de Trabalho, n. 69, 2020.

BARBOSA, L. et al. "Youth, consumption and citizenship: the Brazilian case", in The struggle to belong: dealing with diversity in 21st century urban settings - RC21 Conference. Amsterdam, 2011, pp. 1-16. 
CASTRO, H. C. O.; CAPISTRANO, D. "Cultura política pós-consenso de Washington: o conceito de cultura cívica e a mudança política na América Latina". Revista Debates, v. 2, n. 1, 2008.

CASTRO, H. C. O. et al. "Is self-expression chic? Globalisation, value change and convergence in Latin America". Revista Latinoamericana de Opinión Pública, v. 9, n. 2, 2020, pp. 29-48.

CASTRO, H. C. O.; RANINCHESKI, S.; CAPISTRANO, D. "O conteúdo da globalização para os latino-americanos: uma análise a partir da Pesquisa Mundial de Valores - WVS". Temas y Debates, 2015.

CONVERSE, P. E. "The nature of belief systems in mass publics (1964)". Critical Review, v. 18, n. 1-3, 2006, pp. 1-74.

COSTA, A. M.; RIZZOTTO, M. L. F.; LOBATO, L. de V. C. "Na pandemia da covid-19, o Brasil enxerga o SUS". SCiELO Brasil, 2020.

COUTO, E. S.; COUTO, E. S.; CRUZ, I. de M. P. "\# fiqueemcasa: educação na pandemia da covid-19". Interfaces Científicas-Educação, v. 8, n. 3, 2020, pp. 200-17.

DA SILVA, J. M.; VENTURA, D. D. F. L. "Between science and populism: the Brazilian response to COVID-19 from the perspective of the legal determinants of Global Health". Revista de Direito Internacional, v. 17, n. 2, 2020.

ESAIASSON, P. et al. "How the coronavirus crisis affects citizen trust in institutions and in unknown others: evidence from 'the Swedish experiment'". European Journal of Political Research, 2020, pp. 1-13.

FACCHINI, L. A. "Covid-19: nocaute do neoliberalismo? Será possível fortalecer os princípios históricos do SUS e da APS em meio à pandemia?". APS em Revista, v. 2, n. 1, 2020, pp. 3-10.

FERRAZ, L. M. R. "Saúde e política na crise da covid-19: apontamentos sobre a pandemia na imprensa brasileira". Revista Eletrônica de Comunicação, Informação e Inovação em Saúde, v. 14, n. 2, 2020.

GONZÁLEZ, R. S.; BAQUERO, M.; GROHMANN, L. G. M. "¿Nueva derecha o vino viejo en odres nuevos? La trayectoria conservadora en Brasil en el último siglo". Política y Sociedad, v. 57, n. 3, 2020, pp. 647-70.

INGLEHART, R. "After postmaterialism: an essay on China, Russia and the United States: A comment". Canadian Journal of Sociology, v. 41, n. 2, 2016, pp. 213-22.

INGLEHART, R. Modernization and postmodernization in 43 societies. Princeton University Press, 1997.

INGLEHART, R. The silent revolution: changing values and political styles in advanced industrial society. Princeton, Princeton University Press, 1977.

INGLEHART, R.; WELZEL, C. Modernization, cultural change and democracy: the human development sequence. Edinburgh, Cambridge University Press, 2005.

MELLON, J.; BAILEY, J.; PROSSER, C. "How do coronavirus attitudes fit into britain's ideological landscape?". Parliamentary Affairs, 2021, pp. 1-20.

MENÉNDEZ, M. A.; GONZÁLEZ, R. S. Presentación, v. 57, n. 3, 2019.

OLIVEIRA, P.; NERY, T. "O papel da política externa brasileira e da paradiplomacia na resposta ao coronavírus/Brazilian foreign affairs and paradiplomacy role in the Coronavirus response efforts". Mural Internacional, v. 12, 2021.

PINHEIRO-MACHADO, R. et al. Brasil em transe: bolsonarismo, nova direita e desdemocratização. Oficina Raquel, 2019. 
PIRES, R. R. C. "Os efeitos sobre grupos sociais e territórios vulnerabilizados das medidas de enfrentamento à crise sanitária da covid-19: propostas para o aperfeiçoamento da ação pública TT". Nota Técnica/IPEA Diest, v. 33, 2020, p. 11.

PRIETO-URSÚA, M.; JÓDAR, R. "Finding meaning in hell. The role of meaning, religiosity and spirituality in posttraumatic growth during the coronavirus crisis in Spain". Frontiers in Psychology, v. 11, 2020, pp. 1-8.

REESKENS, T. et al. "Stability or change of public opinion and values during the coronavirus crisis? Exploring Dutch longitudinal panel data". European Societies, v. 23, n. S1, 2021, pp. S153-S171.

REI, F. "La pandemia de la covid-19 y la acción de los nuevos actores internacionales en el ámbito de la governanza global y de la paradiplomacia". Revista de Direito da Cidade, v. 13, n. 2, 2021, pp. 753-74.

RIBEIRO, E. A.; BORBA, J.; HANSEN, J. R. "Participação on-line e off-line no Brasil: relações e condicionantes". Revista do Serviço Público, v. 67, n. 4, 2016, pp. 497-524.

RIBEIRO, E.; BORBA, J. "Participação e pós-materialismo na América Latina”. Opinião Pública, v. 16, 2010, pp. 28-64.

ROTHSTEIN, M. A. "The Coronavirus pandemic: public health and American values". Journal of Law, Medicine and Ethics, v. 48, n. 2, 2020, pp. 354-9.

SANTOS, F.; TANSCHEIT, T. "Quando velhos atores saem de cena: a ascensão da nova direita política no Brasil”. Colombia Internacional, n. 99, 2019, pp. 151-86.

SANTOS-PINTO, C. D. B.; MIRANDA, E. S.; OSORIO-DE-CASTRO, C. G. S. "O 'kit-covid' e o Programa Farmácia Popular do Brasil". Cadernos de Saúde Pública, v. 37, 2021.

TANIGUCHI, M. "A time machine: new evidence of post-materialist value change". International Political Science Review, v. 27, n. 4, 2006, pp. 405-25.

THE LANCET. "Covid-19 in Brazil: 'so what?'". The Lancet, v. 395, n. 10235, 2020, p. 1461.

TORMOS, R. "Postmaterialist values and adult political learning. Changing Intracohort Values in Western Europe". Revista Española de Investigaciones Sociológicas (REIS), v. 140, n. 1, 2012, pp. 201-28.

TORMOS, R. The rhythm of modernization: how values change over time. Boston, Brill, 2019. USLANER, E. M. The moral foundations of trust. Cambridge, Cambridge University Press, 2002.

VENTURA, D. D. F. L.; COSTA BUENO, F. T. "De líder a paria de la salud global: Brasil como laboratorio del 'neoliberalismo epidemiológico' ante la Covid-19". Foro Internacional, 2021, pp. 427-67.

VIZCARRA CASTILLO, S. I.; SANTOS, D. D. O.; DE CASTRO, H. C. O. "Fake news no contexto da pandemia de covid-19: considerações a partir da cultura política". Rizoma, v. 8, n. 1, 2020, pp. 165-84.

WERNECK, G. L.; CARVALHO, M. S. "A pandemia de covid-19 no Brasil: crônica de uma crise sanitária anunciada". Cadernos de Saúde Pública, v. 36, n. 5, 2020, pp. 5-8. 